\title{
ANTISYNTHETASE SYNDROME WITHOUT CLINICAL EVIDENCE OF PULMONARY INVOLVEMENT
}

\begin{abstract}
lane Tamara Dondé1,*, Carlos Augusto Rodrigues Padilha ${ }^{1}$, Danielly Dantas Pimentel ${ }^{1}$, Gustavo Roberto Lourenço ${ }^{1}$, Juliana de
\end{abstract} Jesus Boscolo ${ }^{1}$, Maria Juliana da Silva Almeida ${ }^{1}$, Patrícia Milani de Moraes ${ }^{1}$, Renata Vaz de Oliveira ${ }^{1}$, Taísa Morete da Silva ${ }^{1}$

1.Faculdade de Medicina de São José do Rio Preto, São José do Rio Preto (SP), Brazil.

*Corresponding author: ianetamara@hotmail.com

\section{BACKGROUND}

Antisynthetase syndrome (AS) is characterized by myositis, Raynaud's phenomenon, fever, interstitial lung disease, mechanic's hands and arthropathy associated with the presence of antibodies against tRNA synthetase, especially anti-Jo-1. It can occur in up to $1 / 3$ of patients with dermatomyositis or polymyositis. The average age at diagnosis with anti-Jo-1-positive AS syndrome is 50 years, with a female predominance of 2:1. Interstitial lung disease (ILD) is a common feature in AS with an incidence of 70-90\%. We report a case of antisynthetase syndrome in young woman, but without clinical evidence of pulmonary involvement.

\section{CASE REPORT}

A 33-year-old woman with no previous comorbidities, significant family history of rheumatoid arthritis, who presented with a 2-year history of polyarthralgia, myalgia, and weight loss. There was also a history of a weakness of the muscles of the shoulder and pelvic girdle at the same period. Physical examination confirmed muscle weakness of the left shoulder (4/5) and left pelvic (4/5) girdles and showed erythematous plaques over knuckles suggestive of Gottron's papules. It also revealed swelling and tenderness of right wrist, second right metacarpophalangeal joint, few proximal interphalangeal joints and bilateral knee. Raynaud's phenomenon and periungual erythema were noted, as well as subcutaneous nodules on the extensor surfaces of the forearms. Investigations done showed a normal full blood count and renal function, with elevated levels of CRP (1.2 mg/dL), ESR (45 mm/h), AST (78 IU/L), ALT (32 IU/L), CPK (1794 UI/L), aldolase (37 U/L), LDH (736 U/L). There was presence of polyclonal gammopathy in serum protein electrophoresis. Rheumatology workup demonstrated a negative antinuclear antibody (ANA), anti-CCP $(31.3 \mathrm{U} / \mathrm{mL})$ and rheumatoid factor $(16.4 \mathrm{UI} / \mathrm{mL}$ ). Serological tests revealed a positive anti-aminoacyl-transfer RNA synthetase (anti-ARS) result with positivity for anti-Jo-1 antibodies $(83 \mathrm{U} / \mathrm{mL})$. Hands X-ray and high-resolution chest CT scan were normal. Nailfold capillaroscopy showed scleroderma pattern. The clinical features in conjunction with elevated CPK and aldolase, anti-Jo-1 positivity and the nailfold capillaroscopy findings lead to a presumptive diagnosis of antisynthetase syndrome. The patient was commenced on oral corticosteroids with clinical improvement.

\section{CONCLUSION}

Antisynthetase syndrome is a rare chronic systemic autoimmune disease and it can have varied clinical presentations. Interstitial lung disease is the most frequently occurring manifestation and it is an important prognostic indicator. Anti-Jo- 1 was the first antiARS to be discovered, the most detected and the strongest predictor of ILD. We report a case of antisynthetase syndrome without clinical evidence of pulmonary involvement.

\section{KEYWORDS}

Dermatomyositis, Antisynthetase syndrome, Interstitial lung disease. 